\title{
Computer and Robotic Model of External Fixation System for Fracture Treatment
}

\author{
Yoon Hyuk Kim and Soon-Geul Lee \\ School of Advanced Technology, Kyung Hee University, 1 Sochon-ni, \\ Kihung-eop, Yongin-shi, Kyongki-do, 449-701, Korea \\ yoonhkimakhu.ac.kr
}

\begin{abstract}
A computer graphic model combined with a robotic model of a unilateral external fixation system for a fractured tibia was developed to simulate the pre-operative planning of bone fracture and deformity correction by adjustment of fixator joints. An inverse kinematics analysis algorithm was developed to quantify the necessary rotations and translations at each joint of the fixator. The graphic model was developed using commercial CAD software, and the robotic model was developed to have same configurations. The accuracy of each motor in the robotic model was successfully tested. For a given rotational deformity with a fracture gap, the simulation results of the fracture reduction process were fully matched with the robotic execution. The presented models and techniques can be used for knowledge-based fracture treatment and bone deformity correction using external fixators.
\end{abstract}

\section{Introduction}

External fixation has been widely used in bone fracture fixation and deformity correction [1]. External fixation has a distinct advantage in that it allows adjustment of the bone deformity intra-operatively in one setting or post-operatively through gradual distraction of the bone segments at the osteotomy site [2,3]. Furthermore, it allows ongoing monitoring and correction of bone segment alignment to avoid residual deformity in rotation and translation. In addition to stabilization and adjustment, external fixation also provides mechanical stimulation at the fracture site through the elastic properties of the pins and the joints.

However, it is often difficult to accurately correct a given deformity because optimal adjustment is determined by the trained eye of a clinician, and not by biomechanical data, which is the necessary for precise adjustment of the fixator joints [4]. In addition, the length of radiographic exposure to the clinicians during the procedure can be prolonged since bone segment alignment is evaluated by empirical methods and trial-and-error. It would be very useful to determine the precise fixator joint adjustments needed to accurately execute the correction plan. The plan could also be validated pre-operatively using computer simulation techniques [5].

\footnotetext{
* This study was supported in part by Res. Inst. of Med. Instr. \& Reh. Eng., Kosef, Korea.
} 
Robotic systems are used in various industries to provide stable and accurate manipulation of objects. Such robotic systems may also be applied to the accurate and efficient execution of fracture reduction and deformity correction. To the author's knowledge, there have been no studies thus far attempting to apply robotic systems towards fracture treatment execution.

The objective of this study was to develop an open-link type robotic external fixator model combined with a corresponding computer model of a unilateral external fixator to simulate the required adjustments necessary for optimal bone fracture reduction and deformity correction. The inverse kinematics analysis algorithm was developed to determine the necessary fixator joint adjustments under given bone deformities and fixator application configurations. A computer graphic model of both the fixator and fractured tibia was developed to visualize and validate the analysis result. A robotic model with the same configuration as the computer graphic model was developed and tested. These models and simulation techniques assure precise execution of the desired deformity correction.

\section{Materials and Methods}

\subsection{Development of Robot and Computer Model of External Fixation System}

A serial link type robot model based on a clinically popular unilateral external fixator for fracture fixation and bone deformity correction (Dynafix ${ }^{\circledR}$, EBImedical, USA) was developed in this study. The fixator robot model is composed of four pins inserted into the bones, two pin clamps, four revolute joints, and two sliding joints, respectively (Fig. 1a). In the robot model, AI-motors system ${ }^{\circledR}$ (Megarobotics, Korea) was used to construct the revolute joints, and rack-and-pinion gears were added to the revolute joints to represent the prismatic joints. The AI-motor system is a moduletype motor system that has both a control and link unit. It is relatively light, small in size, and very inexpensive, making it ideal for construction of the preliminary robot model. The robot system is controlled by Matlab ${ }^{\circledR}$ (Mathworks, USA) with the accuracy of $1.3^{\circ}$ in rotation and $0.154 \mathrm{~mm}$ in translation, according to the manufacturer's report. Geometric dimensions of the robot model were measured and the joint types were defined to facilitate the inverse kinematic analysis.

The computer graphic model of a transversely fractured tibia at the diaphyseal area was modeled from CT data (Visible Human data, NIH, USA). The graphic model of the external fixator was developed and the bone-fixator system assembled using commercial CAD software, SolidWorks ${ }^{\mathrm{TM}}$ (Solidworks, MA, USA). Then, computer simulation of the deformity correction process was performed using commercial animation software (3D Studion Max ${ }^{\circledR}$, Autodesk Inc., USA) in order to visualize the computed results of joint rotation and translation required to achieve the desired fracture reduction process and evaluate the performance of the robot model. In the computer model, the geometric dimensions and configurations of the fixator, including the pin insertions, are the same as those of the robot model. 


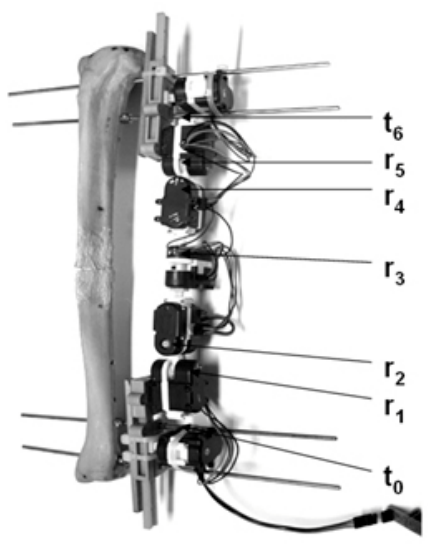

(a)

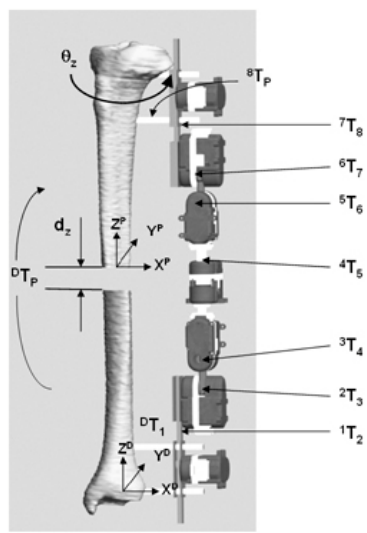

(b)

Fig. 1. The robot, (a), and the computer graphic, (b), models of the Dynafix ${ }^{\circledR}$ external fixator system (EBImedical, USA)

\subsection{Inverse Kinematics Analysis Algorithm}

The mathematical model of the motion of each link of the bone-fixator system can be represented as a open-link serial manipulator system interconnected by five revolute and two prismatic joints, thus having 7 degrees-of-freedom (DOF).

A $4 \times 4$ homogeneous transformation matrix was utilized to express the kinematic loop equations of the fixator-tibia system in order to define six bone deformity parameters at the fracture site [5]. The rotational sequences at the fracture site follows the $x-y^{\prime}-z "$ Euler-angle system, and the global coordinate system was fixed to the distal segment of the tibia. All local joint coordinate systems were defined identically to the global coordinate system. In the mathematical model, ${ }^{\mathrm{D}} \mathbf{T}_{\mathrm{P}}$ is the transformation matrix from the proximal tibial segment to the distal segment, was expressed by the matrix or chain equation shown below:

$$
{ }^{\mathrm{D}} \mathbf{T}_{\mathrm{p}}={ }^{\mathrm{D}} \mathbf{T}_{1} \cdot{ }^{1} \mathbf{T}_{2} \cdot{ }^{2} \mathbf{T}_{3} \cdot{ }^{3} \mathbf{T}_{4} \cdot{ }^{4} \mathbf{T}_{5}{ }^{5} \mathbf{T}_{6}{ }^{6} \mathbf{T}_{7} \cdot{ }^{7} \mathbf{T}_{8} \cdot{ }^{8} \mathbf{T}_{\mathrm{P}} .
$$

${ }^{\mathrm{D}} \mathbf{T}_{1}$ and ${ }^{8} \mathbf{T}_{\mathrm{P}}$ represent the rigid body translations of the local coordinate systems between the bone segments and the pin clamps (Fig. 1b). The matrices ${ }^{2} \mathbf{T}_{3},{ }^{3} \mathbf{T}_{4},{ }^{5} \mathbf{T}_{6}$ and ${ }^{6} \mathbf{T}_{7}$ are pure rotations at the revolute joints. ${ }^{4} \mathbf{T}_{5}$ represents the axial rotation at the central body. ${ }^{1} \mathbf{T}_{2}$ and ${ }^{7} \mathbf{T}_{8}$ represent translation of the prismatic joints at the pin clamps. After substituting the unknown fracture $\left({ }^{\mathrm{D}} \mathbf{T}_{\mathrm{P}}\right)$ and fixator geometric parameters into the transformation matrices, the seven unknown joint variables, $\mathbf{t}_{\mathbf{0}}, \mathbf{r}_{\mathbf{1}}, \mathbf{r}_{\mathbf{2}}, \mathbf{r}_{3}$, $\mathbf{r}_{5}, \mathbf{r}_{5}$ and $\mathbf{t}_{6}$, can be determined by solving Eq. (1) (Fig. 1a). The resulting systems of non-linear equations were solved using the nonlinear least square optimization method (MATLAB $^{\mathrm{TM}}$, Mathworks, MA, USA) [5]. 


\subsection{Validation of the Models and Simulation of Fracture Treatment}

To validate the developed computer and robot models of bone-fixator system, in the first, the accuracy of each revolute and prismatic joint was tested. The accuracy of a joint was defined by the difference between the arbitrarily applied joint values in the control program and the encoder values of the joint motor after joint movement.

A correction of $45^{\circ}$ of rotational deformity combined with $20 \mathrm{~mm}$ of fracture gap was then simulated using the computer graphic animation and the robotic execution. Different adjustment options, such as sequential adjustment with small increments and simultaneous adjustment, were tested in order to fully illustrate the 3-D fixator adjustability and the ability of the simulation to achieve accurate alignment correction during treatment. Based on analysis results and the corresponding graphic animation, the deformity correction planning process can be clearly visualized for evaluation of the analysis results.

\section{Results}

\subsection{Accuracy Test}

Table 1 shows the error values between the input joint values and the measured encoder values from the motors for the robot model. In the table, the maximum error of the all five revolute joints was $1.3^{\circ}$, and these values coincided with the manufacturer specification. The maximum error of the prismatic joints at each pin clamps was 0.14 $\mathrm{mm}$, since the rack-and-pinion gear system used in this model translated $1.3^{\circ}$ of rotational error to $0.14 \mathrm{~mm}$.

\subsection{Simulation of Fracture Reduction Process}

In the case of the correction of a $45^{\circ}$ rotational deformity with $20 \mathrm{~mm}$ of fracture gap, the solution of the fixator joint variables was obtained for the developed fixator system, with the following result: $\left(\mathbf{r}_{1}=17^{\circ}, \mathbf{r}_{2}=-7^{\circ}, \mathbf{r}_{\mathbf{3}}=-43^{\circ}, \mathbf{r}_{4}=-7^{\circ}, \mathbf{r}_{\mathbf{5}}=-17^{\circ}, \mathbf{t}_{\mathbf{0}}=\right.$ $\left.17 \mathrm{~mm}, \mathbf{t}_{\mathbf{6}}=12 \mathrm{~mm}\right)$. Upper and lower bounds imposed on the rotational and prismatic fixator joints based on the fixator design constraints improve the solution convergence.

Based on the joint values calculated from the inverse kinematics analysis, the robotic execution of the fracture treatment to correct the given deformity was performed (Fig. 2a). The adjustment sequence of the robotic execution was from the distal revolute joint, $\mathbf{r}_{\mathbf{1}}$, to the proximal joints, $\mathbf{r}_{\mathbf{5}}$, then the two prismatic joints, $\mathbf{t}_{\mathbf{0}}$ and $\mathbf{t}_{\mathbf{6}}$.

Upon implementing the resulting inverse kinematics analysis values into the fixator joint variables, the computer graphic simulation indicated achievement of perfect reduction the given deformity (Fig. $2 b$ and $2 c$ ). Two types of fracture reduction processes were simulated for testing clinical options. In Fig. $2 b$, we experimented with new adjustment techniques, by first inserting the pins into the appropriate positions of the bone segments and assembling the fixator frames according to the analysis results, 
then readjusting all the joints to their neutral positions. In Fig. 2c, the conventional approach executed by clinicians was simulated, by first constructing the fixator frames in their neutral positions, then changing the fixator joints according to the analysis results. The resulting actions on the bone segments in both cases should perfectly reduce the given deformity.

In order to examine the accuracy of the robotic execution, the reverse adjustment procedure was performed from the neutral positions to generate the same deformity, which was a $45^{\circ}$ rotational deformity with $20 \mathrm{~mm}$ of gap. As shown in Fig. 3, the final rotations and the gap generated after the robotic execution was $43^{\circ}$ and $20 \mathrm{~mm}$, respectively. This error came from the low stiffness of the AI motors used in this study.

Table 1. The rotational errors of the revolute joints and the translational errors of prismatic joints in the robot model of the external fixation system

\begin{tabular}{rlcccc}
\hline \hline \multirow{2}{*}{$\begin{array}{c}\text { Input } \\
(\mathrm{deg})\end{array}$} & \multicolumn{5}{c}{$\begin{array}{c}\text { Rotational Error } \\
(\mathrm{deg})\end{array}$} \\
\cline { 2 - 6 } & $\mathrm{R} 1$ & $\mathrm{R} 2$ & $\mathrm{R} 3$ & $\mathrm{R} 4$ & $\mathrm{R} 5$ \\
\hline-52.00 & 0.00 & 1.30 & 0.00 & 1.30 & 1.30 \\
-31.20 & 0.00 & 0.00 & 0.00 & 1.30 & 1.30 \\
-10.40 & 0.00 & 0.00 & 0.00 & 0.00 & 0.00 \\
0.00 & 1.30 & 1.30 & 0.00 & 0.00 & 0.00 \\
10.40 & 1.30 & 1.30 & 0.00 & 0.00 & 0.00 \\
31.20 & 1.30 & 1.30 & 0.00 & 0.00 & 0.00 \\
52.00 & 1.30 & 1.30 & 0.00 & 0.00 & 1.30 \\
\hline \hline
\end{tabular}

\begin{tabular}{rcr}
\hline \hline \multirow{2}{*}{$\begin{array}{c}\text { Input } \\
(\mathrm{mm})\end{array}$} & \multicolumn{2}{c}{ Trans. Error (mm) } \\
\cline { 2 - 3 } & \multicolumn{1}{c}{ T0 } & \multicolumn{1}{c}{ T6 } \\
\hline 0.00 & 0.00 & 0.15 \\
4.63 & 0.00 & 0.00 \\
9.25 & 0.00 & 0.15 \\
13.88 & 0.15 & 0.15 \\
18.50 & 0.00 & 0.00 \\
23.13 & 0.15 & 0.00 \\
27.75 & 0.15 & 0.15 \\
\hline \hline
\end{tabular}

\section{Discussion and Conclusions}

External fixation is commonly used to stabilize long bone segments following fracture or for bone lengthening $[1,2,3]$. This surgical treatment has several advantages such as adjustment capability, elastic fixation, easy removal from the bones, and mechanical stimulation. External fixation methods also provide different surgical options such as gradual correction of the residual deformity at the fracture site can be accomplished by rotating and translating the fixator joints gradually on an adjustment plan. Despite the many advantages of external fixation, it has not been favored as the treatment of choice even when clinical indications are favorable for such treatment because of improper pre-operative planning and inaccurate execution from the surgical planning [4]. In order to obtain long-term good clinical results, computer-assisted 3-D preoperative planning and precise execution of external fixation is necessary $[4,6,7]$.

Some limitations of the current robot model have been found, such as oscillation of the fixator frames during adjustment and correction errors of the fracture sites. These limitations come from the low torque and low stiffness of the AI-motors used in this study, since this model was developed primarily to demonstrate the feasibility of robotic execution techniques for external fixation. These problems could be eliminated easily by adopting high-torque and high stiffness low velocity motors for the robot system. 
(a)
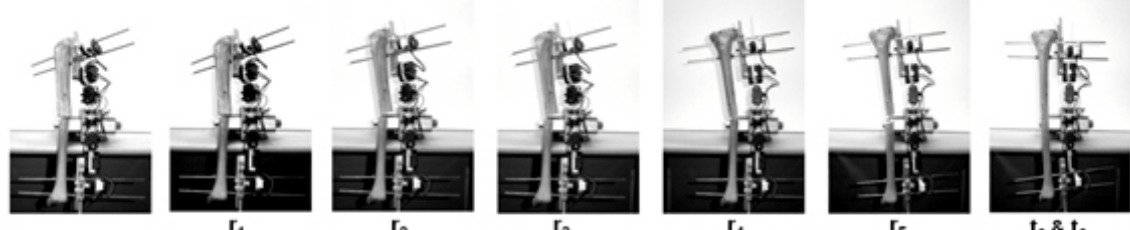

(b)
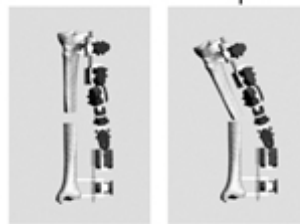

$r_{2}$

$r_{5}$

$\mathrm{t}_{0} \& \mathrm{t}_{6}$
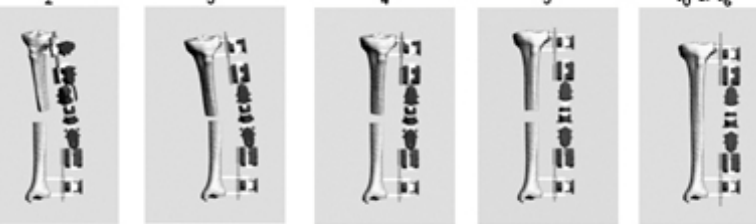

(c)
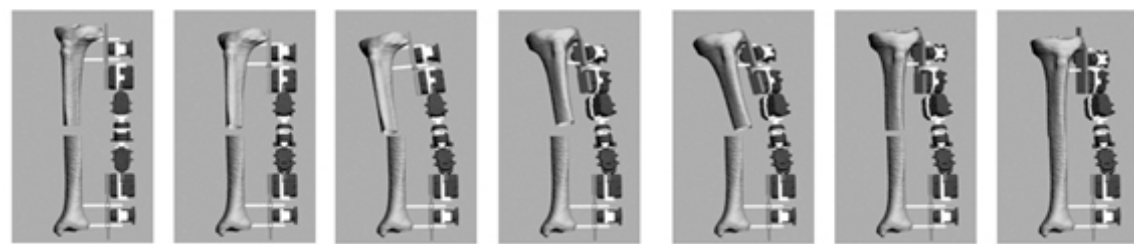

Fig. 2. The computer simulation and robotic execution of $45^{\circ}$ of external rotation with a $20 \mathrm{~mm}$ gap by sequential adjustment from the proximal to distal revolute joints following the two end prismatic joints. (a) Robotic executions of initial fixator system with adjusted positions of the joints. (b) Computer simulation of fixator system with joints initially in adjusted positions. (c) Computer simulation of fixator system with joints initially in neutral positions
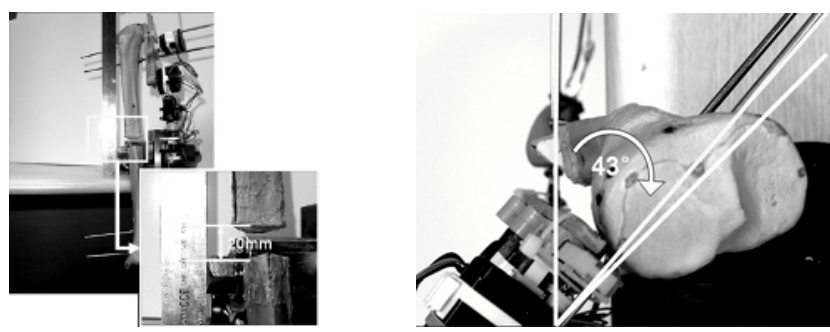

Fig. 3. The results of rotation and gap after execution of robot model of the external fixation system for a $45^{\circ}$ of external rotation with a $20 \mathrm{~mm}$

In summary, this paper presents the development of a computer graphic model with the robotic model of an external fixation system for fracture treatment and deformity correction. Using the developed inverse kinematics analysis, the required amount of the rotation and translation of the fixator joints are determined to reduce given fracture deformities. Based on these calculated joint values, the developed robot model of the bone-fixator system demonstrates the execution of the adjustments for a given fracture deformity. In addition, the computer graphic model developed in this study can accurately simulate the adjustment process to visualize and evaluate the pre-operative 
planning procedure. In the future, the combined tools could be used for executing knowledge-based computer-aided fracture treatment, enhancing clinical performance and facilitating changes in the design configuration for the external fixator.

\section{References}

1. Chao E.Y., Hein T.J.: Mechanical performance of the standard Orthofix ${ }^{\circledR}$ external fixator. Orthopedics 11 (1988) 1057-1069.

2. Paley, D., Tetsworth, K.: Mechanical axis deviation of the lower limbs; Preoperative planning of uniapical deformities of the tibia and femur. Clin. Orthop. Related Res. 280 (1992) 48-64

3. Choi, I.H., Chung, C.Y., Cho ,T.J., Park, S.S.: Correction of genu recurvatum by the Ilizarov method. J. Bone Joint Surg. 81-B (1999) 769-774

4. Hsu, R.W.W., Himeno, S., Coventry, M.B., Chao, E.Y.S.: Normal axial alignment of the lower extremities and load-bearing distribution at the Knee. Clin. Orthop. Related Res. 255 (1990) 215-227

5. Kim, Y.H., Inoue, N., Chao, E.Y.S.: Kinematic simulation of fracture reduction and bone deformity correction under unilateral external fixation. J. Biomech. (2002) 1047-1058

6. Ellis, R.E., Tso, C.Y., Rudan, J.F., Harrison, M.M.: A surgical planning and guidance system for high tibial osteotomy. Comput. Aided Surg. 4 (1999) 264-274

7. Chao, E.Y.S., Sim, F.H.: Computer-aided preoperative planning in Knee osteotomy. The Iowa Orthop. J. 15 (1995) 4-18

8. Larsen, S.T., Magyar, G., Onsten, I., Ryd, L., Lindstrand, A.: Upper tibial valgus osteotomy using a dynamic external fixator. J. Bone Joint Surg. 80-B (1998) 295-297

9. Chao E.Y.S., Rim K., Smidt G.I., Johnston RC.: The application of 4 X4 matrix method to the correction of the measurements of hip joint rotations. J. of Biomech. 3 (1970) 459-471 